\title{
Pretreatment of Macrophages with the Combination of IFN- $\gamma$ and IL-12 Induces Resistance to Leishmania major at the Early Phase of Infection
}

\author{
Haruko OTA $^{1)}$, Yasuhiro TAKASHIMA ${ }^{2)}$, Yoshitsugu MATSUMOTO ${ }^{3)}$, Yoshihiro HAYASHI ${ }^{1)}$ and \\ Yasunobu MATSUMOTO ${ }^{1) *}$ \\ ${ }^{1)}$ Department of Global Animal Resource Science, Graduate School of Agricultural and Life Sciences, The University of Tokyo, 1-1-1 \\ Yayoi, Bunkyo-ku, Tokyo 113-8657, 2)Department of Veterinary Parasitological Diseases, Faculty of Applied Biological Sciences, Gifu \\ University, 1-1 Yanagito, Gifu 501-1193 and ${ }^{3)}$ Department of Molecular Immunology, Graduate School of Agricultural and Life \\ Sciences, The University of Tokyo, 1-1-1 Yayoi, Bunkyo-ku, Tokyo 113-8657, Japan
}

(Received 25 December 2007/Accepted 7 February 2008)

\begin{abstract}
Interferon (IFN)- $\gamma$ is essential but not sufficient to control leishmaniasis. It is known that IFN- $\gamma$ is one of the major macrophage-activating cytokines, and the activated macrophages are a principal source of interleukin (IL)-12, which induces autocrine macrophage activation. In this study, the combined effect of IFN- $\gamma$ and IL-12 on the susceptibility of macrophages to Leishmania major infection was evaluated. Macrophages pretreated with IFN- $\gamma$ and/or IL-12 were infected with the parasites. Four hr post-infection (p.i.), the levels of infection and parasite load in the macrophages treated with the combination of IFN- $\gamma$ and IL-12 (IFN- $\gamma /$ IL-12) were significantly lower than those in the nontreated cells. However, the macrophages treated with either IFN- $\gamma$ or IL-12 did not show resistance to $L$. major infection. In addition, $72 \mathrm{hr}$ p.i., the IFN- $\gamma / \mathrm{IL}-12$-treated and IFN- $\gamma$-treated macrophages showed significantly lower levels of infection and parasite load than the nontreated cells, and higher levels of resistance was observed in the IFN- $\gamma / \mathrm{IL}-12$-treated macrophages than in the IFN- $\gamma$-treated macrophages. Although IFN- $\gamma / \mathrm{IL}-12$ treatment of macrophages prior to the infection led to the induction of resistance, as described above, this resistance was not induced when these cytokines and the parasites were added simultaneously to the macrophage culture. These results suggest that IFN- $\gamma / \mathrm{IL}-12$ treatment prior to the infection restricts the early phase of the infection. KEY WORDS: interferon- $\gamma$, interleukin-12, Leishmania, macrophage.
\end{abstract}

Studies on Leishmania as well as Toxoplasma and Mycobacterium have been largely responsible for the characterization of the Th1/Th2 paradigm of healing/nonhealing associated with intracellular infection $[3,4,17]$. Interferon (IFN)- $\gamma$ was identified as a cytokine required for not only Th1 cell differentiation [18] but also macrophage activation to suppress the activity of Leishmania infecting the macrophages [5]. In an experimental murine leishmaniasis model, it was reported that the susceptibility of mice to Leishmania infection is due to the lack of Th1 responses related to IFN- $\gamma$ production and the development of interleukin (IL)-4-dominated Th2 responses [6]. However, despite the importance of IFN- $\gamma$ in the development of resistance to Leishmania infection, it was reported that the administration of exogenous IFN- $\gamma$ does not protect susceptible BALB/c mice from L. major infection [15]. In addition, IFN- $\gamma$ mRNA was detected in the infected skin lesions of not only resistant $\mathrm{C} 57 \mathrm{BL} / 6$ mice but also susceptible BALB/c mice [21]. These reports suggest that IFN- $\gamma$ alone is not sufficient to control L. major and that additional factor(s) would be required for the development of protective immunity to $L$. major. IL-12 is known as a cytokine involved in Th1 cell differentiation, and it stimulates NK cells to produce IFN- $\gamma$ $[8,19,20]$. Moreover, IL-12 induces autocrine macrophage

\footnotetext{
* Correspondence to: Matsumoto, Y., Department of Global Animal Resource Science, Graduate School of Agricultural and Life Sciences, The University of Tokyo, 1-1-1, Yayoi, Bunkyo-ku, Tokyo 113-8657, Japan.

e-mail: aymatsu@mail.ecc.u-tokyo.ac.jp
}

activation and consequent IL-12 and IFN- $\gamma$ production from the activated macrophages $[10,12]$. Previously, it was reported that continuous treatment of $L$. major-infected mice with IL-12 suppressed disease progression [7], and that IL-12-deficient mice were more susceptible to $L$. major infection than wild-type control mice [11]. These results indicate the importance of IL-12 in the development of resistance to $L$. major infection.

Leishmania infects phagocytic cells such as macrophages and dendritic cells in the mammalian host [2]. IFN- $\gamma$ is one of the principle macrophage-activating cytokines, and the activated macrophages are one of the principal sources of IL-12 [14]. Therefore, it is expected that IFN- $\gamma / \mathrm{IL}-12$ are directly related to the behavior of Leishmania in infected macrophages.

In this study, we focused on the effect of IFN- $\gamma$ and IL-12 on L. major infection in macrophages and demonstrated that these cytokines restrict the L. major infection during the early phase, i.e., before inception of parasite proliferation.

\section{MATERIALS AND METHODS}

Mice: Six-week-old female BALB/c mice were obtained from Japan SLC, Inc. (Shizuoka, Japan). Animal experiments were carried out under the strict guidance of the Animal Research Committee in accordance with the Guidelines on Animal Experiments at The University of Tokyo.

Parasites: The L. major PM2 strain (MHOM/UZ/91/ PM2) was kindly provided by Dr. Margarita Strelkova 
(Martsinovsky Institute of Medical Parasitology and Tropical Medicine, Moscow, Russia). Promastigotes were cultured in 199 medium (Nissui Pharmaceutical Co., Ltd, Tokyo, Japan) containing 10\% heat-inactivated fetal calf serum (FCS; Thermo Electron Corp., Melbourne, Australia) and $20 \mathrm{mM}$ HEPES (MP Biomedicals Inc., Aurora, OH) at $25^{\circ} \mathrm{C}$. Infectious-stage metacyclic promastigotes were used for this study.

Macrophages: Resident mouse peritoneal macrophages were harvested from the peritoneal cavity by lavage with 5 $\mathrm{m} l$ of ice-cold phosphate-buffered saline (PBS). For each experiment, cells isolated from three or four mice were pooled. Macrophages were centrifuged and resuspended in ice-cold PBS. After additional 2 washes with ice-cold PBS, the cells were suspended at a density of $2.5 \times 10^{5} / \mathrm{ml}$ in RPMI 1640 medium (Sigma) containing 10\% heat-inactivated FCS and $20 \mu \mathrm{g} / \mathrm{m} l$ gentamicin (Schering-Plough, Kenilworth, NJ). The cells were then cultured on a 16-well chamber slide (Nalge Nunc, Rochester, NY) at $37^{\circ} \mathrm{C}$ with $5 \% \mathrm{CO}_{2}$ in air. After incubation for $2 \mathrm{hr}$, the slides were washed three times in the medium to remove the unattached cells, and the remaining (attached) cells were used as the macrophage monolayer.

In vitro infection: Prior to inducing the infection, the macrophage monolayer, prepared as described above, was cultured for $15 \mathrm{hr}$ in the presence of $1 \mathrm{ng} / \mathrm{ml}$ recombinant IFN$\gamma(\mathrm{rIFN}-\gamma)$ (BD Bioscience, San Diego, CA) and/or $1 n \mathrm{~g} / \mathrm{m} l$ rIL-12 (R\&D systems, Minneapolis, MN) at $37^{\circ} \mathrm{C}$ with $5 \%$ $\mathrm{CO}_{2}$ in air. L. major promastigotes were suspended at a concentration of $5 \times 10^{6}$ cells $/ \mathrm{ml}$ in the media containing the same cytokines used for the pretreatment of the macrophage monolayer. The suspension of L. major promastigotes was then added to the macrophage monolayer in each well at a ratio of five parasites per macrophage. Four hr postinfection (p.i.), the cultures were washed to remove the unbound parasites, and fresh media containing the same cytokines that were used during the preculture and infection procedures were added into each well. As a control condition, macrophages were cultured and infected with promastigotes by following the process described above except that the cells were cultured in a cytokine-deficient medium for all the procedures. Four or $72 \mathrm{hr}$ p.i., the wells were washed with PBS, and the cells were fixed with $100 \%$ methanol and stained with Giemsa solution. Using light microscopy, 300 macrophages per well were observed, and the number of infected macrophages and the number of intracellular parasites were counted. To examine the effect of cytokines added to the culture after the parasitic infection, the harvested macrophages were incubated for $15 \mathrm{hr}$ in a cytokinedeficient medium and infected with only promastigotes, using the above-described procedure. After washing out the unbound parasites, fresh media with or without $1 \mathrm{ng} / \mathrm{m} l$ rIFN- $\gamma$ and/or $1 n \mathrm{~g} / \mathrm{m} l \mathrm{rIL}-12$ were added to each well. After 4 or $72 \mathrm{hr}$ p.i., the number of infected macrophages and the number of intracellular parasites were counted as described above. The following three evaluation criteria were examined in the present study: (1) infection rate, calcu- lated as the percentage of infected macrophages to all macrophages; (2) the number of parasites per 100 macrophages; and (3) parasite load, which is the number of parasites per infected macrophage. The results are representative of more than three separate experiments.

Statistical analysis: Data are expressed as mean \pm S.D. One-way ANOVA with post hoc analysis with the TurkeyKramer test was used to compare groups.

\section{RESULTS}

Effect of IFN- $\gamma$ and IL-12 on the susceptibility of macrophages to L. major infection: To investigate the effect of IFN- $\gamma$ and IL-12 on the susceptibility of macrophages to $L$. major infection, peritoneal macrophages pretreated with IFN- $\gamma$ and/or IL-12 were infected with the parasites.

Four hr p.i., the levels of infection rate and the number of parasites per 100 macrophages in the macrophages treated with IFN- $\gamma / \mathrm{IL}-12$ were significantly lower than those in the nontreated cells (Fig. 1A and 1B). The number of macrophages infected with more than one parasite was lower in the treated macrophages than in the nontreated cells (Fig. 1C). However, when the macrophages were treated with either IFN- $\gamma$ or IL-12, the cells did not exhibit resistance to the infection (Fig. 1).

At the late phase of infection, i.e., $72 \mathrm{hr}$ p.i., the IFN- $\gamma / \mathrm{IL}-$
(A)

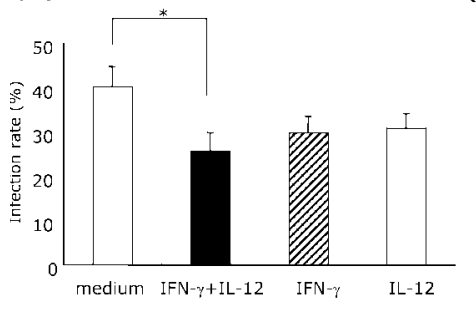

(C)
(B)

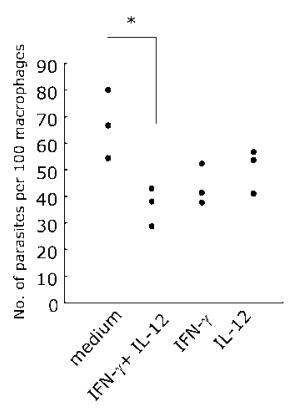

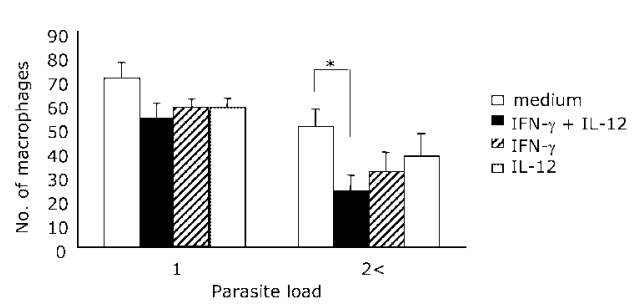

Fig. 1. Induction of resistance to L. major at the early phase of infection in cytokine-treated macrophages. The effect of cytokine to induce resistance to L. major in macrophages was determined by the infection rate (A), the number of parasites per 100 macrophages (B), and the parasite load (C). Prior to the infection, the macrophages were cultured in the cytokine-deficient medium (open), medium containing IFN- $\gamma / \mathrm{IL}-12$ ( $1 \mathrm{ng} / \mathrm{m} l$ each, filled), $1 n \mathrm{~g} / \mathrm{m} l \mathrm{IFN}-\gamma$ (hatched), or $1 n \mathrm{~g} / \mathrm{m} l \mathrm{IL}-12$ (grey), as described in the Materials and Methods. Four hr after the addition of the parasites, the numbers of infected macrophages and intracellular parasites were evaluated. All data are presented as mean \pm S.D. ${ }^{*} \mathrm{p}<0.05$. 
(A)

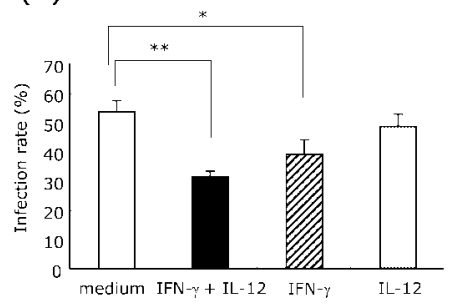

(B)

(C)

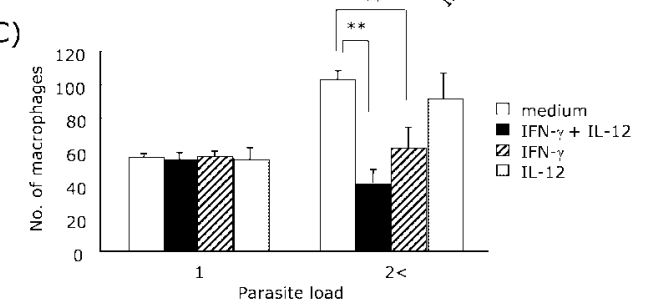

Fig. 2. Induction of resistance to L. major at the late phase of infection by cytokine treatment of macrophages. The effect of cytokines to induce resistance to L. major in macrophages was determined by the infection rate (A), the number of parasites per 100 macrophages (B), and the parasite load (C). Prior to the infection, the macrophages were cultured in the cytokine-deficient medium (open), medium containing IFN- $\gamma / \mathrm{IL}-12(1 \mathrm{ng} / \mathrm{ml}$ each, filled), $1 n \mathrm{~g} / \mathrm{m} l \mathrm{IFN}-\gamma$ (hatched), or $1 n \mathrm{~g} / \mathrm{m} l \mathrm{IL}-12$ (grey), as described in the Materials and Methods. Seventy-two hr after the infection, the numbers of infected macrophages and intracellular parasites were evaluated. All data are presented as mean \pm S.D. * $\mathrm{p}<0.05, * * \mathrm{P}<0.01$.

12-treated macrophages showed significantly lower $L$. major infection rates and numbers of parasites per 100 macrophages than the nontreated control cells (Fig. 2A and 2B). The number of macrophages infected with more than 1 parasite was also lower in the treated cells than in the nontreated cells. Consistent with a previous report [5], the IFN$\gamma$-treated macrophages also showed considerably lower infection levels and parasite load than the nontreated cells; however, the resistance was marked in the IFN- $\gamma /$ IL-12treated macrophages (Fig. 2). The macrophages treated with IL-12 alone did not display any resistance to the parasite infection (Fig. 2). These results suggest that in the macrophages, IFN- $\gamma / \mathrm{IL}-12$ induced the resistance to L. major infection at the early phase of infection.

The optimum time for IFN- $\gamma / I L-12$ treatment of macrophages for the control of L. major infection: The fact that IFN$\gamma / \mathrm{IL}-12$ treatment of the macrophages induced resistance to L. major infection at the early phase of infection (Figs. 1 and 2) prompted us to examine whether treatment with these cytokines prior to infection induces resistance to the infection. As shown in Fig. 3, the treatment of macrophages with IFN- $\gamma /$ IL-12 prior to infection significantly reduced the infection rate and the number of parasites per 100 macrophages $4 \mathrm{hr}$ p.i. (Fig. 3A and 3B). On the other hand, when the treatment was commenced immediately after the addition of parasites, induction of the resistance to L. major infection
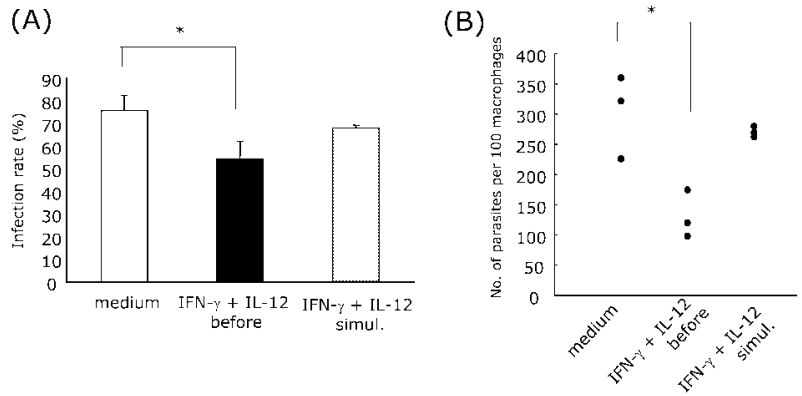

Fig. 3. Inhibition of L. major infection at the early phase of infection by IFN- $\gamma / \mathrm{IL}-12$ treatment prior to the infection. The effect of IFN- $\gamma / \mathrm{IL}-12$ to induce resistance to L. major in macrophages was determined by the infection rate (A) and the number of parasites per 100 macrophages (B). The macrophages were treated with IFN- $\gamma / \mathrm{IL}-12$ prior to the infection $(1 \mathrm{ng} / \mathrm{m} l$, each; filled) or simultaneously with the addition of the parasites (grey), as described in the Materials and Methods. As a control condition, the macrophages were cultured in the cytokine-deficient medium (open) and infected with the parasites. Four hr after the infection, the numbers of infected macrophages and intracellular parasites were evaluated. All data are presented as mean \pm S.D. $* \mathrm{p}<0.05$.
(A)

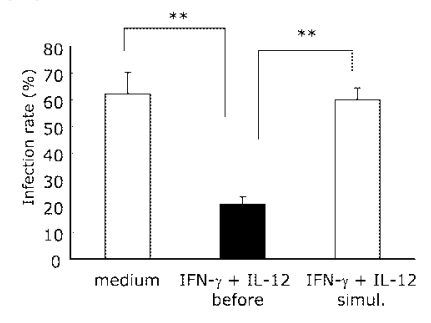

(B)

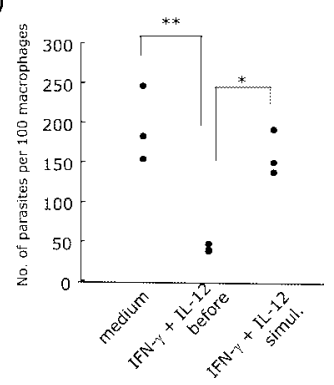

Fig. 4. Inhibition of L. major infection at the late phase of infection by IFN- $\gamma / \mathrm{IL}-12$ treatment prior to the infection. The effect of IFN- $\gamma / \mathrm{IL}-12$ to induce resistance to L. major in macrophages was determined by the infection rate (A) and the number of parasites per 100 macrophages (B). The macrophages were treated with IFN- $\gamma / \mathrm{IL}-12$ prior to the infection ( $1 \mathrm{ng} / \mathrm{m} l$, each; filled) or simultaneously with the addition of the parasites (grey), as described in the Materials and Methods. As a control condition, the macrophages were cultured in a cytokine-deficient medium (open) and infected with the parasites. Seventy-two hr after the infection, the numbers of infected macrophages and intracellular parasites were evaluated. All data are presented as mean \pm S.D. $* \mathrm{p}<0.05, * * \mathrm{P}<0.01$.

was not observed (Fig. 3). There was no reduction in the infection rate and the number of intracellular parasites at 72 hr p.i. when the macrophages were treated with IFN- $\gamma /$ IL-12 and the parasite simultaneously (Fig. 4).

\section{DISCUSSION}

In this study, we investigated the effect of IFN- $\gamma$ and IL12 treatment of the macrophages on the resistance to $L$. major infection. Four hr p.i., statistically significant reduc- 
tion in both infection rate and parasite load was observed when the macrophages were treated with IFN- $\gamma / \mathrm{IL}-12$ prior to the infection (Figs. 1 and 3). The importance of pretreatment with IFN- $\gamma / \mathrm{IL}-12$ in reducing the intracellular parasites suggests that the effect is mediated by the induction of the production of several proteins and/or modulation of their expression levels. Green et al. demonstrated that nitrogen oxide (NO) produced by the action of inducible NO synthetase (iNOS), which is upregulated by IFN- $\gamma$ treatment to macrophages, functions to reduce the number of infected $L$. major $72 \mathrm{hr}$ p.i. [5]. It is thus possible that $\mathrm{NO}$ also plays an important role in reducing the parasite number during an infection. However, there was no difference in the NO levels between the IFN- $\gamma / \mathrm{IL}-12$-treated and nontreated macrophages $4 \mathrm{hr}$ p.i. (unpublished observation). Based on this and other observations, it is suggested that IFN- $\gamma /$ IL-12 treatment of the macrophages prior to the infection induced resistance to $L$. major infection at the early phase in an NOindependent manner.

The proliferation of $L$. major in the infected macrophages might have not initiated until $4 \mathrm{hr}$ p.i. Thus, IFN- $/$ /IL-12 probably induces some inhibition of parasite binding to host macrophages or alteration in invasion as the resistant mechanisms. It has been reported that several cytokines, including IFN- $\gamma$, regulate the expression levels of macrophage surface receptor molecules such as mannose receptor [9] and complement receptor 3 [16], which function as macrophage receptors for Leishmania [1, 12]. It is possible that IFN- $\gamma /$ IL-12 also regulates the expressions of these (or other) receptor molecules on macrophages. Moreover, IFN$\gamma / \mathrm{IL}-12$ treatment to macrophages prior to the infection may affect their intracellular functions such as establishment of parasitophorous vacuole or fusion of the vacuole and lysosome. The results of this study indicate the need and importance of a further study on the possible IFN- $\gamma /$ IL-12-induced regulation underlying the binding or invasion of Leishmania to macrophages.

Treatment with IFN- $\gamma /$ IL-12 induced considerably higher levels of resistance in the macrophages than the treatment with IFN- $\gamma$ alone (Figs. 2 and 4). Interestingly, when the cells were treated with the cytokines prior to the infection, the IFN- $\gamma /$ IL-12-treated macrophages produced greater levels of NO than IFN- $\gamma$-treated macrophages $72 \mathrm{hr}$ p.i. (unpublished observation). It is certain that NO plays an important role in controlling the intracellular parasite. However, the results indicated the involvement of additional mechanisms in inducing resistance to L. major infection in macrophages.

In the animals vaccinated against or resistant to Leishmania infection, tissue macrophages around the infected cells might be exposed to IFN- $\gamma$ and IL-12 secreted from the activated macrophages and probably acquire resistance to parasite binding or invasion at the site of infection. Further, we believe that the parasites that could not infect the macrophages are killed by the complement-mediated pathway, and that resistance to parasite binding or invasion is one of the important defense mechanisms.
ACKNOWLEDGMENTS. This study was supported in part by the 21st Century COE Program "Biodiversity and Ecosystem Restoration Research Project" and the Grant-inaid for Scientific Research (B) (16380198), Exploratory Research (19658113), and International Scientific Research (A) (19256001) from the Ministry of Education, Culture, Sports, Science and Technology, and Health Labor Sciences Research Grant, Research on HIV/AIDS from the Ministry of Health, Labor and Welfare of Japan.

\section{REFERENCES}

1. Akivov, O.E., Kasuboski, R.E., Carter, C.R. and McDowell, M.A. 2007. The role of mannose receptor during experimental leishmanaiasis. J. Leukoc. Biol. 81: 1188-1196.

2. Alexander, J., Satoskar, A.R. and Russell, D.G. 1999. Leishmania species: models of intracellular parasitism. J. Cell Sci. 112: 2993-3002.

3. Flynn, J.L., Chan, J., Triebold, K.J., Dalton, D.K., Stewart, T.A. and Bloom, B.R. 1993. An essential role for interferon $\gamma$ in resistance to Mycobacterium tuberculosis infection. J. Exp. Med. 178: 2249-2254.

4. Gazzinelli, R.T., Wysocka, M., Hayashi, S., Denkers, E.Y., Hieny, S., Caspar, P., Trinchieri, G. and Sher, A. 1994. Parasite-induced IL-12 stimulates early IFN- $\gamma$ synthesis and resistance during acute infection with Toxoplasma gondii. J. Immunol. 153: 2533-2543.

5. Green, S.J., Crawford, R.M., Hockmeyer, J.T., Meltzer, M.S. and Nacy, C.A. 1990. Leishmania major amastigotes initiate the L-arginine-dependent killing mechanism in IFN- $\gamma$-stimulated macrophages by induction of tumor necrosis factor- $\alpha$. $J$. Immunol. 145: 4290-4297.

6. Heinzel, F.P., Sadick, M.D, Holaday, B.J., Coffman, R.L. and Locksley, R.M. 1989. Reciprocal expression of interferon- $\gamma$ or interleukin-4 during the resolution or progression of murine leishmaniasis. Evidence for expansion of distinct helper T-cell subsets. J. Exp.Med. 169: 59-72.

7. Heinzel, F.P., Schoenhaut, D.S., Rerko, R.M., Rosser, L.E. and Gately, M.K. 1993. Recombinant Interleukin 12 cures mice infected with Leishmania major. J. Exp. Med. 177: 1505-1509.

8. Kobayashi, M., Fitz, L., Ryan, M., Hewick, R.M., Clark, S.C., Chan, S., Loudon, R., Sherman, F., Perussia, B. and Trinchieri, G. 1989. Identification and purification of natural killer cell stimulatory factor (NKSF), a cytokine with multiple biologic effects on human lymphocytes. J. Exp. Med. 170: 827-845.

9. Martinez-Pomares, L., Reid, D.M., Brown, G.D., Taylor, P.R., Stillion, R.J., Linehan, S.A., Zamze, S., Gordon, S. and Wong, S.Y.C. 2003. Analysis of mannose receptor regulation by IL-4, IL-10, and proteolytic processing using novel monoclonal antibodies. J. Leukoc. Immunol. 73: 604-613.

10. Marzio, P.D., Puddu, P., Conti, L., Belardelli, F. and Gessani, S. 1994. Interferon- $\gamma$ upregulates its own gene expression in mouse peritoneal macrophages. J. Exp. Med. 179: 1731-1736.

11. Mattner, F., Padova, K.D. and Alber, G. 1997. Interleukin-12 is indispensable for protective immunity against Leishmania major. Infect. Immun. 65: 4378-4383.

12. Mosser, D.M. and Edelson, P.J. 1985. The mouse macrophage receptor for $\mathrm{C} 3 \mathrm{bi}$ (CR3) is a major mechanism in the phagocytsis of Leishmania promastigotes. J. Immunol. 135: 2785-2789.

13. Munder, M., Mallo, M., Eichmann, K. and Modolell, M. 1998. Murine macrophages secrete Interferon $\gamma$ upon combined stimulation with Interleukin (IL)-12 and IL18: a novel pathway of 
autocrine macrophage activation. J. Exp. Med. 187: 21032108.

14. Puddu, P., Fantuzzi, L., Borghi, P., Varano, B., Rainaldi, G., Guillemard, E., Malorni, W., Nicaise, P., Wolf, S.F., Belardelli, F. and Gessani, S. 1997. IL-12 induces IFN-gamma expression and secretion in mouse peritoneal macrophages. $J$. Immunol. 159: 3490-3497.

15. Sadick, M.D., Heinzel, F.P., Holaday, B.J., Pu, R.T., Dawkins, R.S. and Locksle, R.M. 1990. Cure of murine leishmaniasis with anti-interleukin 4 monoclonal antibody. Evidence for a $\mathrm{T}$ cell-dependent, interferon gamma-independent mechanism. $J$. Exp. Med. 171: 115-127.

16. Schlesinger, L.S. and Horwitz, M.A. 1991. Phagocytosis of Mycobacterium leprae by human monocyte-derived macrophages is mediated by comprement receptors CR1 (CD35), CR3 (CD11b/CD18), and CR4 (CD11c/CD18) and IFN- $\gamma$ activation inhibits complement receptor function and phagocytosis of this bacterium. J. Immunol. 147: 1983-1994.

17. Scott, P., Natovitz, P., Coffman, R.L., Pearce, E. and Sher, A.
1988. Immunoregulation of cutaneous leishmaniasis. T-cell lines that transfer protective immunity or exacerbation belong to different T-helper subsets and respond to distinct parasite antigens. J. Exp.Med. 168: 1675-1684.

18. Scott, P. 1991. IFN- $\gamma$ modulates the early development of Th1 and Th2 responses in a murine model of cutaneous leishmaniasis. J. Immunol. 147: 3149-3155.

19. Scott, P. 1993. IL-12: initiation cytokine for cell-mediated immunity. Science 260: 496-497.

20. Seder, R.A., Gazzinelli, A., Sher, A. and Paul, W.E. 1993. Interleukin 12 acts directly on $\mathrm{CD}^{+} \mathrm{T}$ cells to enhance priming for interferon $\gamma$ production and diminishes interleukin 4 inhibition of such priming. Proc. Natl. Acad. Sci. U.S.A. 90: 1018810192.

21. Stenger, S., Thuring, H., Rollinghoff, M. and Bogdan, C. 1994. Tissue expression of inducible nitric oxide synthesis is closely associated with resistance to Leishmania major. J. Exp. Med. 180: 783-793. 